\title{
Classification of Mental Tasks from EEG Signals Using Spectral Analysis, PCA and SVM
}

Nikolay N. Neshov, Agata H. Manolova, Ivo R. Draganov, Krasimir T. Tonschev, Ognian L. Boumbarov

Technical University of Sofia, 8 Kl. Ohridskiblvd., 1756 Sofia,Bulgaria
E-mails: $\quad$ nneshov@tu-sofia.bg amanolova@tu-sofia.bg
k_tonchev@tu-sofia.bg olb@tu-sofia.bg

Abstract: Signals provided by the ElectroEncephaloGraphy (EEG) are widely used in Brain-Computer Interface (BCI) applications. They can be further analyzed and used for thinking activity recognition. In this paper we proposed an algorithm that is able to recognize five mental tasks using 6 channel EEG data. The main idea is to separate the raw EEG signals into several frames and compute their spectrums. Next, a second-order derivative of Gaussian is applied to extract features and an optimum Gaussian kernel parameters grid search is performed with the help of cross-validation. The extracted features are further reduced by Principal Component Analysis. The processed data is utilized to train SVM classifier which is used for mental tasks recognition afterwards. The performance of the algorithm is estimated on publically available dataset. In terms of 5 folds cross-validation we obtained an average of $82.7 \%$ recognition rate (accuracy). Additional experiments were conducted using leave-one-out cross-validation where $67.2 \%$ correct classification was reported. Comparison to several state-of-the art methods reveals the advantages of the proposed algorithm.

Keywords: ElectroEncephaloGraphy (EEG), Brain Computer Interface (BCI), Fast Fourier Transform (FFT), Principal Component Analysis (PCA), Support Vector Machine (SVM).

\section{Introduction}

In recent years, the ElectroEncephaloGraphy-based (EEG-based) Brain-Computer Interface (BCI) has become one of the most promising areas of research in computer science and robotics thanks to neurorehabilitation. Neurorehabilitation is a relatively new field and a multi-step system that combines series of therapies from the psychological to occupational, teaching or retraining patients on mobility skills, communication processes and other aspects of that person's daily routine [1]. The main goal of this complex medical process is the recovery of the patient from a 
nervous system injury and the minimization and/or compensation for related functional alterations or disabilities. There are several common medical conditions that are treated by this scientific field, for example: Cerebral palsy, Parkinson's disease, Brain injury, Spinal cord injuries, Multiple sclerosis, Stroke, Post-polio syndrome and Muscular Dystrophy. What these conditions have in common is the presence of damaged communication between the source of movement (brain, specific motor cortex) and the actuator of movement (muscles). The human nervous system is a highly complex, extremely versatile and dynamic neural network with countless inputs and outputs that govern the entire existence of the human being. So if the doctors, rehabilitators or the medical personnel in general want to achieve any functional improvement, they need to be able to repair or substitute that part of the neural network that is not functional in part or in total anymore. Some of their goals may be to permanently replace with an appropriate device or artificial limb, or repair by retraining and re-learning, the impaired functionality. In either case one needs to study the relations between inputs and outputs of the system in order to achieve better patient rehabilitation.

A lot of research from different field experts is put into replacing or regaining the lost functionality with the use of emerging technologies such as robotics, computer vision, virtual reality and brain-computer interfaces for enhancing the disabled user's independence. The EEG is very important to understand the interaction of the different brain areas, study the effect of the abovementioned diseases on the neurological processes and build a BCI that can help paraplegic individuals [2]. The BCI will use the signals from neuronal activity in the brain to interface them with a computer. Thanks to EEG measurement many researchers are able to develop new technologies and therapies allowing the assessment of the resulting changes in the patient's brain [3] during their therapy sessions. Thus, the development of practical BCIs for disabled people using EEG signals should allow them to use all their existing mental and muscle functionalities as control possibilities in the system $[4,5]$. This method had proved to be effective in helping patients with severe motor deficits to control remote devices such as computer cursor, artificial limbs or even moving a wheelchair. Various profiles can be built and different control strategies could be applied, depending on the condition of the patient.

The rest of the paper is organized as follows: In the next section we provide a brief state-of-the-art review of some methods used for mental task recognition based on EEG signals. In Section 3 we present the content of the EEG Database [6] that we used to perform our investigations. In Section 4 we illustrate and explain the proposed algorithm. In Section 5 we give the experimental results. Finally, in Section 6, the paper ends with conclusions and discussions.

\section{Related work}

Determining the user's activities is not only necessary for neurorehabilitation but according to [7] it is central for ubiquitous computing. The authors have developed an unobtrusive and lightweight single electrode BCI system that can be used to 
recognize with accuracy between 70-100\% different mental activities such as reading and relaxing with Bayesian networks. An interesting scenario is presented in [8] which deals with feature extraction and classification of horizontal mental task pattern on 1-D cursor movement from EEG signals. In this case three neural network classifiers are used: learning vector quantization, multilayer neural network and probabilistic neural network. Mou st af a and Ga d [9] use Linear Predictive Coding and Discrete Wavelet Transform for compression of EEG channels and feature extraction combined with Support Vector Machines (SVM) for classification of five mental tasks form [6] with an average recognition rate of about $85 \%$. For the same database Hariharan et al. [10] propose the stockwell transform for feature extraction and then compare three classifiers: $k$-means nearest neighbours, linear discriminant analysis and SVM to test the strength of the proposed features.

All cited in this paper works that share the same database contribute their own innovation in the field of mental tasks analysis for different applications either neurorehabilitation and BCI or ubiquitous computing. But it should be mentioned that direct comparison of their results is difficult due to the lack of uniformity in using the number of subjects, performing the types of experiments and presenting the results.

One of the main goals of our work is finding-out an appropriate feature extraction process for better analysing EEG signals. A pool of methods for EEG feature extraction exists in the literature. The Auto-Regressive (AR) model, The Discrete Wavelet Transform (DWT), and the Fast Fourier Transform (FFT) are widely used for such purpose. The AR model is mainly used in signal processing especially for system identification [11]. Usually, in the AR model, the variable of interest is predicted by linear combination of past values of the variable. The feature vector is formed from the coefficients of the model and further used in BCI system [12]. However, the AR model cannot capture transient features from EEG signals [13] and the AR analysis suffers from speed, and hence it is not always applicable in real time applications. DWT represents efficiently EEG signals by decomposing each signal into frequency sub-bands. On the other hand, the window with varying size is the most significant parameter of this method since it ensures the suitable time frequency resolution in all frequency ranges [14]. Hence the choice of suitable window size might be tricky for building high accurate BCI system. In our work we use FFT as preferred method for feature extraction since it provides suitable frequency domain representation of the signal, it is fast, and is one of the most widely used method in EEG analysis.

\section{Database}

In this study, we have used the publically available database, collected by Keirn and Aunon, from the BCI laboratory of Colorado State University [6]. The database contains EEG signals recorded from seven subjects, each of which performed at least five trials of five pre-defined mental tasks (each trial has duration of 10 seconds). 
The mental tasks are:

1. Baseline (relaxing as much as possible).

2. Multiplication (calculating multiplication mentally).

3. Letter-Composing (considering the contents of a letter).

4. Rotation (imagining rotation of a 3-D object).

5. Counting (imagining writing a number in order).

The data were measured by 6 EEG channels and 1 EOG channel (to measure the movement of an eye). The electrodes are placed as shown in Fig. 1 and the measurements are made with reference to electrically linked mastoids, A1 and A2.

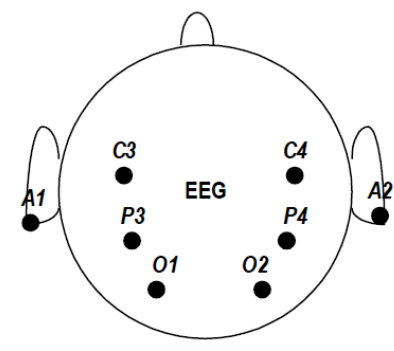

Fig. 1. Electrode placement

The electrodes are connected to a bank of active filters whose band-pass bandwidths are set from 0.1-100 Hz. The data is passed to 12-bit ADC operated at a sampling rate of $250 \mathrm{~Hz}$. Thus, for a given task and a subject - 2,500 samples $(250 \mathrm{~Hz} \times 10 \mathrm{~s})$ per channel are recorded (for the whole trial). The experiments in this study are conducted for each subject independently. Thus, it is suitable to examine the algorithm performance utilizing equal amount of data per person. This allows objective comparison of the results among the subjects. Taking into account that subjects 2 and 7 completed only five trials, the analyses are performed over the EEG signals for the first five trials only for each participant.

\section{Algorithm description}

A general block diagram representing the main steps of the proposed algorithm is shown in Fig. 2.

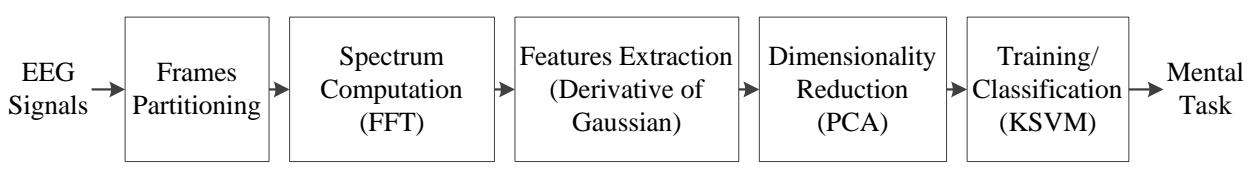

Fig. 2. Main steps of the proposed algorithm for mental tasks classification

The EEG signal from each channel is first normalised to zero mean and variance one. Further it is divided into adjacent frames (each one has duration of 1 second which corresponds to a length of 250 samples). Thus, 10 frames are produced per channel. This process is illustrated on Fig. 3. 


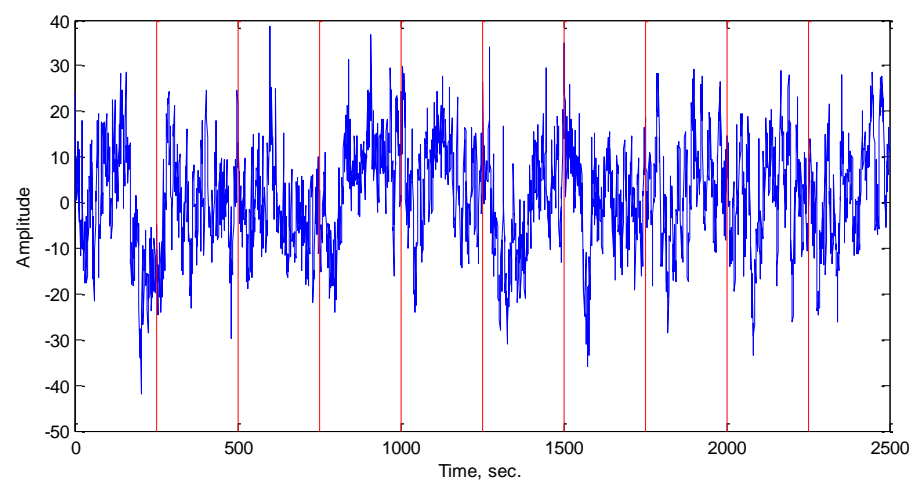

Fig. 3. Frames partitioning of the EEG signal recorded for one channel

The period of $1 \mathrm{~s}$ is chosen as reasonable for practical mental tasks recognition purposes. One can be asked to perform some thinking activity for at least one second before refocusing mind into another direction. Additional tests in our experimental setup showed that the maximum classification performance is reached using comparable time periods. The next step is Fast Fourier Transformation (FFT). The motivation behind the choice of FFT is inspired by the assumption that there is a relation between specific mental task and the distribution of magnitudes of specific group of frequencies of the EEG signals. Considering 250 samples for each frame and 6 channels data we have to compute 6 spectrums at a time. After applying the FFT the resulted spectrum is symmetrical around the zero frequency. Thus, only the half part (i.e., 125 samples) is further used in the next steps. The spectrum representation for one of the frames (obtained for subject 4) is given on Fig. 4.

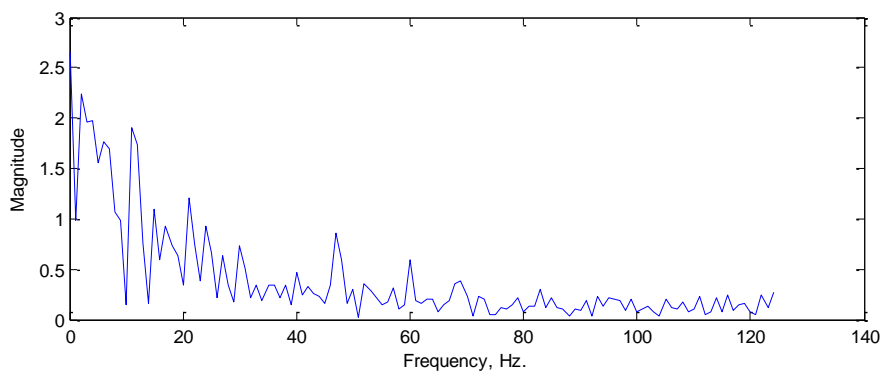

Fig. 4. Result after applying FFT over one frame of EEG signal

The third step is feature extraction. This is essential part for reaching high recognition accuracy. In order to describe the rate of change of spectrum shape we convolved the spectrum using second derivative Gaussian filter of size $s$. The 2nd derivative of Gaussian function can be expressed as follows:

$$
g^{\prime \prime}(x)=\left(\frac{x^{2}}{\sigma^{4}}-\frac{1}{\sigma^{2}}\right) e^{-\frac{x^{2}}{2 \sigma^{2}}} .
$$


The choice of suitable kernel parameters (the filter size $s$ and the standard deviation $\sigma$ ) is very important for getting higher classification accuracy. We evaluated the classification performance for each person (in terms of 5 folds crossvalidation accuracy) using different sets of parameters $(s, \sigma)$. The analyses are presented later-on in Section 5. The results produced by this step are 6 feature vectors per frame (i.e., one feature vector per channel). Each feature vector has 125 dimensional representations. An example illustration of feature bins distribution (obtained for the signal from Fig. 4) is depicted in Fig. 5.

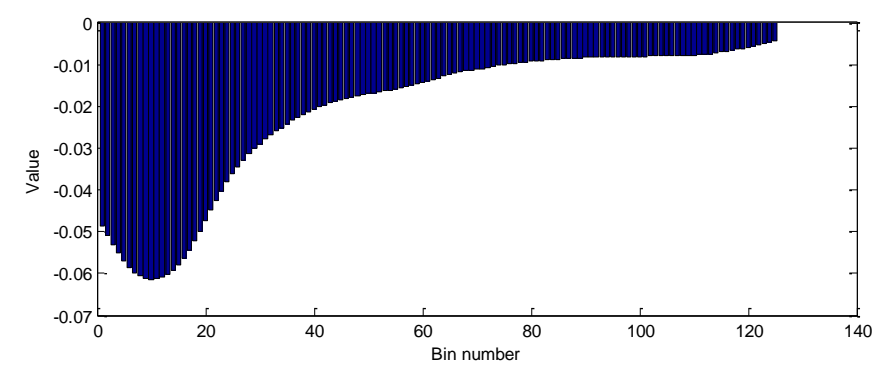

Fig. 5. Example feature vector bins distribution after applying 2nd order derivative of Gaussian filter $(s=40, \sigma=20-$ see Table 1$)$

To form a dataset for analysis, we concatenated all 6 feature vectors in one which generates $6 \times 125=750$ dimensional feature vector. Further, we used Principal Component Analysis (PCA) to reduce the vector's size preserving 99\% of the energy. One of the main advantages of PCA is its ability to reduce the dimensionality without much loss of information. The reduced vector after PCA is then utilized for training Support Vector Machine (SVM) classifier using LibSVM [15]. SVM is supervised learning method, commonly used for regression and classification purposes. It builds a discriminant hyper-plane that maximizes the margins to distinguish the classes. For the binary classification problem, given a training set of dataset-label pairs $\left(x_{i}, y_{i}\right)$, where $i=1, \ldots, N$ and $y_{i}=+1$ or -1 , the SVM solves the following optimization problem:

$$
\min _{w, b, c} \frac{1}{2} w^{\mathrm{T}} w+C \sum_{i=1}^{N} \varepsilon_{i}
$$

subject to: $y_{i}\left(w^{\mathrm{T}} \varphi\left(x_{i}\right)+b\right) \geq 1-\varepsilon_{i}, \varepsilon_{i} \geq 0$.

The training vectors $x_{i}$ can be mapped into a higher dimensional space by the function $\varphi$. In the non-linear cases, the SVM can create decision boundaries with the help of kernel function $K\left(x_{i}, x_{j}\right)=\varphi\left(x_{i}\right)^{\mathrm{T}} \varphi\left(x_{j}\right)$ [16]. In our study we use Radial Basis Function (RBF) where the optimum parameters are found by grid-search. To solve the multi-classification problem we used one-against-one strategy.

Once the SVM model is built (by processing all EEG training datasets) the algorithm can be used in classification/testing mode. In this mode the unknown/testing EEG signals are passed through each of the aforementioned steps. Once the reduced feature vector is produced it is further classified by SVM. 


\section{Experimental results}

\subsection{Finding optimal filter parameters}

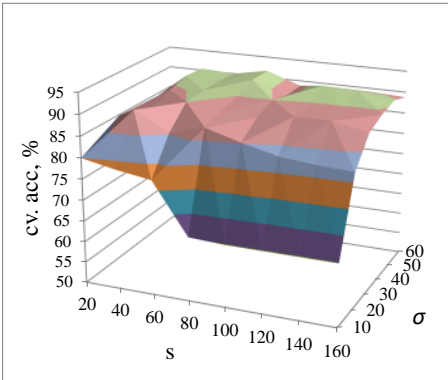

(a) Subject $1, s_{\text {opt }}=120, \sigma_{\text {opt }}=50, A_{\max }=92 \%$

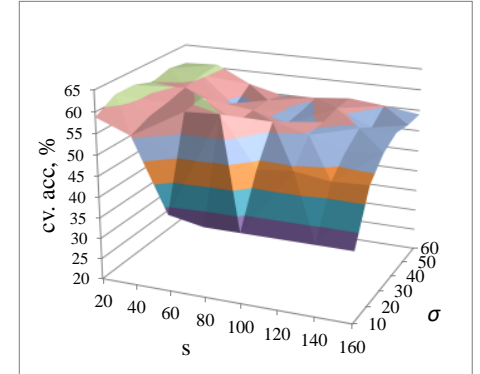

(c) Subject 3, $s_{\text {opt }}=20, \sigma_{\text {opt }}=30, A_{\text {acc }}$ max $=61,6 \%$

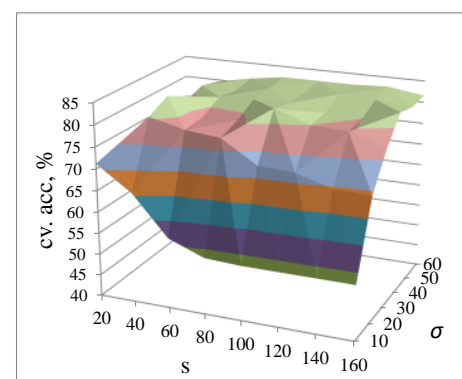

(e) Subject 5, $s_{\text {opt }}=40, \sigma_{\text {opt }}=30, A_{\text {cc }} \max =82,8 \%$

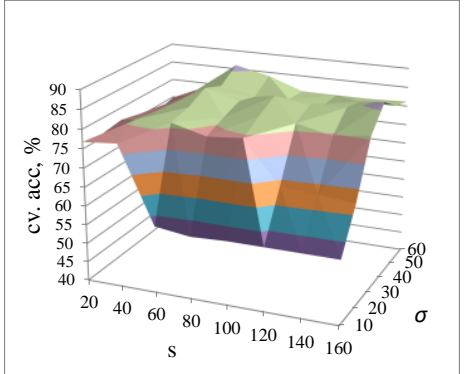

(b) Subject 2, $s_{\mathrm{opt}}=160, \sigma_{\mathrm{opt}}=40, \mathrm{Acc}_{\max }=86,4 \%$

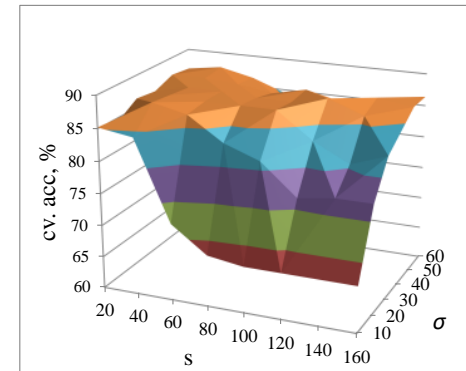

(d) Subject 4, $s_{\text {opt }}=40, \sigma_{\text {opt }}=20, A_{\text {cc }}$ max $=88 \%$

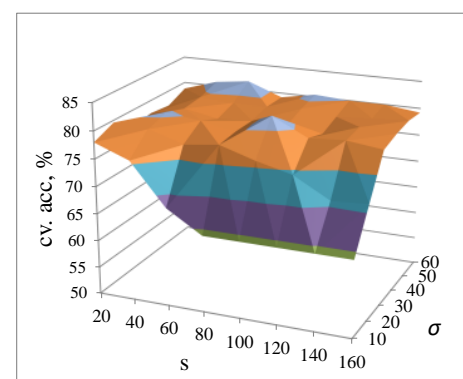

(f) Subject 6, $s_{\text {opt }}=98100, \sigma_{\mathrm{opt}}=30, \mathrm{Acc}_{\max }=82 \%$

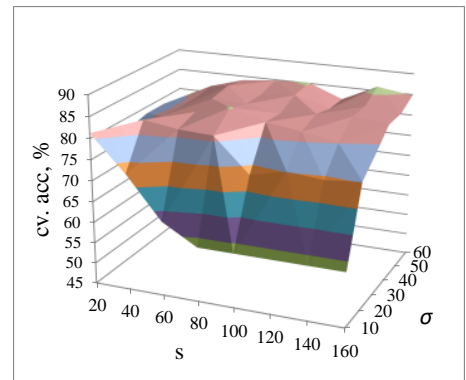

(g) Subject 7, $s_{\text {opt }}=140, \sigma_{\text {opt }}=60, A_{\text {cc }} \max =86 \%$

Fig. 6. Optimum filter parameter grid search via 5-fold cross-validation accuracy for each subject 
As mentioned earlier we applied a grid search to find-out the optimal filter kernel parameters for each subject individually. Especially, the size of the kernel $s$ is in the range from 20 up to 120 with an increment of 20. The parameter $\sigma$ is between 10 and 60 and the increment is 10 . The results of these analyses are illustrated in Fig. 6a-g.

It can be seen that the pair of kernel parameters (optimum size $s_{\text {opt }}$ and optimum standard deviation $\sigma_{\mathrm{opt}}$ ) which gives the maximum cross-validation Accuracy $\left(\mathrm{Acc}_{\max }\right)$ varies among the different persons. This means that for real applications of the algorithm, the optimum parameters should be found for a given user prior building the SVM model. In general (considering all subjects) from Fig. 6, it can be observed that the lowest accuracy performances are reported for the smallest standard deviations and the influence of variation of $\sigma$ over the recognition accuracy is higher than that of $s$. The results from these investigations are presented in Table 1. The maximum accuracy is obtained for Subject $1(92 \%)$ and the minimum - for Subject $3(61.6 \%)$. The recognition rate is $82.7 \%$ on average. The optimum parameters found are further used for the experiments reported in the next subsection.

Table 1. 5-fold cross-validation accuracy obtained with the optimum filter parameters

\begin{tabular}{|c|c|c|c|}
\hline Subject & $S_{\text {opt }}$ & $\sigma_{\text {opt }}$ & Accuracy, $\%$ \\
\hline 1 & 120 & 50 & 92 \\
\hline 2 & 160 & 40 & 86,4 \\
\hline 3 & 20 & 30 & 61,6 \\
\hline 4 & 40 & 20 & 88 \\
\hline 5 & 40 & 30 & 82.8 \\
\hline 6 & 100 & 30 & 82 \\
\hline 7 & 140 & 60 & 86 \\
\hline
\end{tabular}

\subsection{Performance evaluation}

In order to investigate the recognition performance of our algorithm in more realistic situations, we applied leave-one-trial-out cross-validation strategy for each subject individually. This means that at a time we used four of all five trials, available for a given subject, as a training data and the remaining one - as a testing data and calculate the recognition accuracy. Further we repeat the same procedure four times, each time considering different trial set for testing (and the remaining trails for training) and then the classification accuracy is evaluated based on the average over the five trials. This experimental setup makes it possible to perform a deep and realistic analysis of the algorithm performance since it represents as close as possible to the real environment scenario where one can be asked to train the system in several trials and further utilizes it in recognition mode. It should be noted that the authors of the most of the state-of-the-art papers do not separate their training/testing sets by trials but perform cross-validation randomly using training and testing samples from a specific trial. We calculate the average recognition accuracy for all seven subjects using the first five trials. Since each trial contains 10 datasets (for a specific task) we have a total of 50 datasets per task. This gives 50 datasets per task $\times 5$ tasks $=250$ datasets for training/testing per person. 
Table 2. Confusion matrices (in number of datasets) per tasks and subjects obtained by leave-one trial out cross-validation (the best results are underlined)

\begin{tabular}{|c|c|c|c|c|c|c|}
\hline Task & \multicolumn{6}{|c|}{ Predicted tasks } \\
\hline \multirow{6}{*}{ 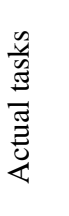 } & & B & M & LC & $\mathrm{R}$ & $\mathrm{C}$ \\
\hline & $\bar{B}$ & 44 & 5 & 1 & 0 & 0 \\
\hline & $\mathrm{M}$ & 11 & 38 & 1 & 0 & 0 \\
\hline & $\mathrm{LC}$ & 0 & 0 & 50 & 0 & 0 \\
\hline & $\mathrm{R}$ & 0 & 0 & 0 & 39 & 11 \\
\hline & $\mathrm{C}$ & 0 & 0 & 0 & 14 & 36 \\
\hline
\end{tabular}

a. Subject 1

\begin{tabular}{|c|c|c|c|c|c|c|}
\hline Task & \multicolumn{6}{|c|}{ Predicted tasks } \\
\hline \multirow{6}{*}{ 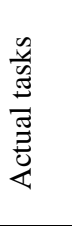 } & & $\mathrm{B}$ & $\mathrm{M}$ & LC & $\mathrm{R}$ & $\mathrm{C}$ \\
\hline & B & 23 & 5 & 10 & 6 & 6 \\
\hline & $\mathrm{M}$ & 9 & 25 & 5 & 4 & 7 \\
\hline & LC & 5 & 3 & \begin{tabular}{|l}
27 \\
\end{tabular} & 10 & 5 \\
\hline & $\mathrm{R}$ & 12 & 3 & $\overline{13}$ & 21 & 1 \\
\hline & $\mathrm{C}$ & 10 & 12 & 6 & 2 & 20 \\
\hline
\end{tabular}

c. Subject 3

\begin{tabular}{|c|c|c|c|c|c|c|}
\hline Task & \multicolumn{6}{|c|}{ Predicted tasks } \\
\hline \multirow{6}{*}{ 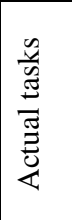 } & & B & $\mathrm{M}$ & $\mathrm{LC}$ & $\mathrm{R}$ & $\mathrm{C}$ \\
\hline & B & 31 & 10 & 6 & 2 & 1 \\
\hline & $\mathrm{M}$ & 6 & 34 & 1 & 1 & 8 \\
\hline & $\mathrm{LC}$ & 6 & $\overline{0}$ & 33 & 0 & 11 \\
\hline & $\bar{R}$ & 4 & 7 & 1 & 31 & 7 \\
\hline & $\mathrm{C}$ & 6 & 12 & 5 & 9 & 18 \\
\hline
\end{tabular}

e. Subject 5

\begin{tabular}{|c|c|c|c|c|c|c|}
\hline Task & & & dic & $\mathrm{d}$ tas & & \\
\hline & & B & $\mathrm{M}$ & LC & $\mathrm{R}$ & $\mathrm{C}$ \\
\hline$\hat{v}$ & B & 37 & 2 & 6 & 0 & 5 \\
\hline$\underset{\Xi}{ \pm}$ & $\mathrm{M}$ & 3 & 43 & 2 & 0 & 2 \\
\hline$\Xi$ & LC & 10 & 0 & 33 & 0 & \begin{tabular}{|l}
7 \\
\end{tabular} \\
\hline$\frac{0}{4}$ & $\mathrm{R}$ & 0 & 4 & 0 & \begin{tabular}{|l|l}
40 \\
\end{tabular} & 6 \\
\hline & $\mathrm{C}$ & 8 & 6 & 5 & \begin{tabular}{|l}
2 \\
\end{tabular} & \begin{tabular}{|l|}
29 \\
\end{tabular} \\
\hline
\end{tabular}

b. Subject 2

\begin{tabular}{|c|c|c|c|c|c|c|}
\hline Task & \multicolumn{6}{|c|}{ Predicted tasks } \\
\hline \multirow{6}{*}{ 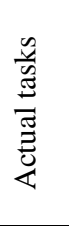 } & & B & $\mathrm{M}$ & LC & $\mathrm{R}$ & $\mathrm{C}$ \\
\hline & B & 40 & 0 & 1 & 9 & 0 \\
\hline & $\mathrm{M}$ & 3 & 26 & 0 & 4 & 17 \\
\hline & LC & 4 & 0 & 39 & 5 & 2 \\
\hline & $\mathrm{R}$ & 12 & 5 & 1 & 32 & 0 \\
\hline & $\mathrm{C}$ & 1 & 1 & 3 & 5 & $\underline{40}$ \\
\hline
\end{tabular}

\begin{tabular}{|c|c|c|c|c|c|c|}
\hline Task & \multicolumn{6}{|c|}{ Predicted tasks } \\
\hline \multirow{6}{*}{$\begin{array}{l}\frac{n}{v} \\
\frac{\tilde{J}}{ \pm} \\
\frac{\pi}{\tilde{Z}} \\
\frac{0}{0}\end{array}$} & & $\mathrm{~B}$ & $\mathrm{M}$ & $\mathrm{LC}$ & $\mathrm{R}$ & $\mathrm{C}$ \\
\hline & $\mathrm{B}$ & 25 & 4 & 13 & 4 & 4 \\
\hline & $\mathrm{M}$ & 3 & 33 & 5 & 9 & 0 \\
\hline & $\mathrm{LC}$ & 10 & 3 & 30 & 2 & 5 \\
\hline & $\mathrm{R}$ & 6 & 7 & 3 & 34 & 0 \\
\hline & $\mathrm{C}$ & 4 & 0 & 3 & 2 & 41 \\
\hline
\end{tabular}

f. Subject 6

\begin{tabular}{|c|c|c|c|c|c|c|}
\hline Task & \multicolumn{6}{|c|}{ Predicted tasks } \\
\hline \multirow{6}{*}{ 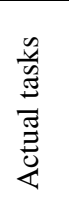 } & & $\mathrm{B}$ & $\mathrm{M}$ & $\mathrm{LC}$ & $\mathrm{R}$ & $\mathrm{C}$ \\
\hline & B & 32 & 5 & 4 & 0 & 9 \\
\hline & $\mathrm{M}$ & 4 & 33 & 0 & 13 & 0 \\
\hline & LC & 13 & 0 & 32 & 0 & 5 \\
\hline & $\mathrm{R}$ & 0 & 15 & 0 & 33 & 2 \\
\hline & $\mathrm{C}$ & 5 & 1 & 2 & 0 & 42 \\
\hline
\end{tabular}

In Table 2 we give the confusion matrices (in number of datasets) obtained for each person. It can be seen that for Subject 1, the Letter-Composing (LC) task is recognized correctly for all 50 datasets. A similar amount of confusion (11 from 50 cases in total) is reported for the pairs Baseline (B) - Multiplication (M) tasks and Rotation (R) - Counting (C). However in the worst case (Counting) 36 from 50 datasets are correctly recognized. Considering the other six subjects we can see disordered distribution of confusion between the certain pairs of tasks. It is also viewed that the best accuracy is reached for different tasks among the different persons. 
Table 3. Confusion matrices (in number of datasets) per tasks and subjects obtained by leave-one trial out cross-validation (the best results are underlined)

\begin{tabular}{|c|c|c|c|c|c|c|c|c|c|}
\hline Task Subject & S1 & $\mathrm{S} 2$ & S3 & $\mathrm{S} 4$ & S5 & S6 & S7 & $\begin{array}{c}\text { Average } \\
\text { accuracy } \\
\text { for each } \\
\text { task } \\
\end{array}$ & $\begin{array}{c}\text { Standard } \\
\text { deviation } \\
\text { for each } \\
\text { task } \\
\end{array}$ \\
\hline Baseline & 80.0 & 63.8 & 39.0 & 66.7 & 58.5 & 52.1 & 59.3 & 59.9 & 12.7 \\
\hline Multiplication & 88.4 & 78.2 & 52.1 & 81.3 & 54.0 & 70.2 & 61.1 & 69.3 & 14.1 \\
\hline $\begin{array}{c}\text { Letter } \\
\text { composing }\end{array}$ & 96.2 & 71.7 & 44.3 & 88.6 & 71.7 & 55.6 & 84.2 & 73.2 & 18.4 \\
\hline Rotation & 73.6 & 95.2 & 48.8 & 58.2 & 72.1 & 66.7 & 71.7 & 69.5 & 14.5 \\
\hline Counting & 76.6 & 59.2 & 51.3 & 67.8 & 40.0 & 82.0 & 72.4 & 64.2 & 14.9 \\
\hline $\begin{array}{c}\text { Average } \\
\text { accuracy for } \\
\text { each subject }\end{array}$ & 82.9 & 73.6 & 47.1 & 72.5 & 59.3 & 65.3 & 69.7 & 67.2 & \\
\hline $\begin{array}{c}\text { Standard } \\
\text { deviation for } \\
\text { each subject }\end{array}$ & 9.2 & 14.1 & 5.5 & 12.2 & 13.4 & 12.0 & 10.1 & & \\
\hline
\end{tabular}

Despite these disordering observations, we see promising results for the Subject 1. Probably this would mean that the success of recognition is hidden in the person's ability to fully concentrate the mind to certain mental task.

To summarize the results of the experiments, we compute the recognition accuracy in two manners: averaging the recognition rates by subjects and by tasks. The results of these investigations are presented in Table 3 and graphically in Fig. 7.

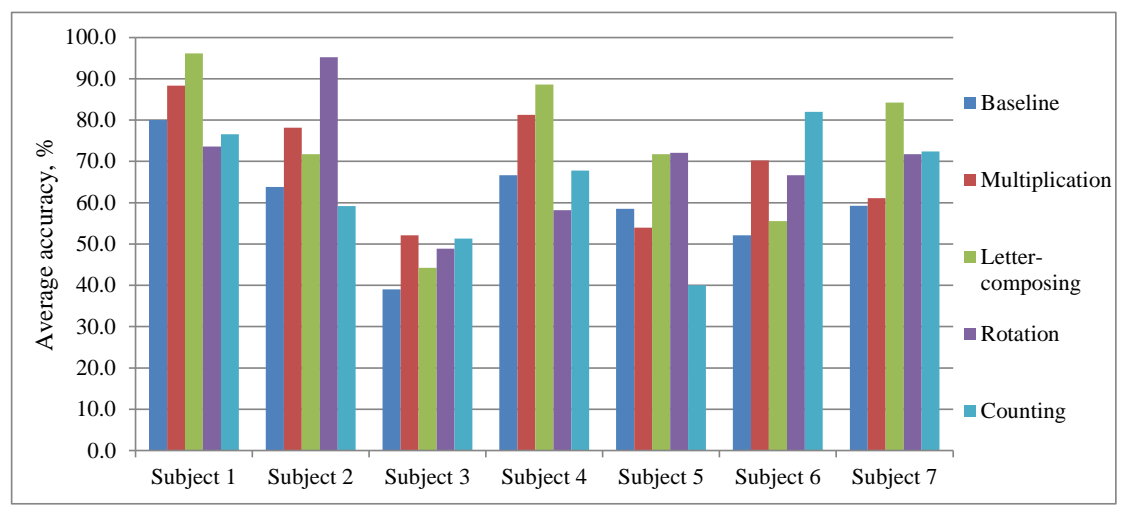

Fig. 7. Graphical representation of average accuracy per task and subject obtained by leave-one trial out cross-validation

Obviously, the best recognition rate is obtained for Subject $1(82.9 \%)$. The worst case is reached for Subject 3 with recognition rate of $47.1 \%$. However, the standard deviation of accuracies does not exceed 13.4. Averaging the results among the different Subjects shows that the highest recognition rate is obtained for the letter-composing task $(73.2 \%)$ and the lowest one - for the Baseline task $(59.3 \%)$. The last observation probably can be explained by the assumption that the full relaxing is difficult to be achieved, which causes misleading brain waves to produce wrong results. 


\subsection{Accuracy comparison to another works}

Comparing the results of our experiments conducted to some of state-of-the-art works shows significant improvements in terms of classification accuracy. For example the algorithm presented in [17] was evaluated on the same brain data and the average recognition rate is only $30 \%$. The proposed method in our paper reaches more than twice higher accuracy (67.2\%). In another work Liang et. al. [18] presented a framework for classification of mental tasks from EEG signals using extreme learning machine. It can be seen that without further post-processing the optimum average classification rate is $56.07 \%$ (which is above $10 \%$ less than the one obtained in our experiments). Even after their suggested post-processing step they reported comparable rate of $67.6 \%$. This shows the potential of our method to be further improved using appropriate post-processing.

\section{Conclusion}

We propose a method and algorithm for mental task recognition using EEG signals. In terms of 5-fold cross-validation accuracy (Section 5.1) we reached $82.7 \%$ correct classification. We conducted additional experiments by using leave-one-trial-out cross-validation strategy, where the average accuracy rate is $62.7 \%$. The experimental results showed that our algorithm provided recognition accuracy higher than the conventional methods. This improvement in the mental tasks classification can provide a better communication pathway between the brain and the machines, which in turn will help to develop more reliable assistive devices such as brain wave controlled wheel chairs, prostatic limbs and smart living environment for patients with brain injuries. We have started working on combining the EEG with EMG signals for estimating and classifying mental and muscle fatigue.

Acknowledgements: This work was supported by contract DFNI I02/1 for research project: "Intelligent man-machine interface for assistive medical systems in improving the independent living of motor disabled users" of the Bulgarian Research Fund of the Ministry of Education and Science.

\section{References}

1. Perez-Marcos, D., M. Solazzi, W. Steptoe, O. Oyekoya, A. Frisoli, T. W e yri ch, A. S t e e d, F. Te c ch i a, M. S l a ter, M. V. S a n ch e z-V ive s. A FullyImmersive Set-Up for Remote Interaction and Neurorehabilitation Based on Virtual Body Ownership. - Frontiersin Neurology, Vol. 3, 2012, No 110.

2. V e ga, C. F., F. J. R. F e r n ánd e z. Recognition of Mental Task with the Analysis of LongRange Temporal Correlations on EEG Brain Oscillation. - In: Proc. of Biosignals and Biorobotics Conference (BRC'12), 2012, ISSNIP.

3. Golomb, M. R., B. C. Mcdonald, S. J. Warden, J. Yonkman, A. J. S a ykin, B. Shirley, M. Huber, B. Rabin, M. Abdelbaky, M. E. Nwos u, M. B a rkatMasih, G. C. Burdea. In-Home Virtual Reality Videogame Telerehabilitation in Adolescents with Hemiplegic Cerebral Palsy. - Arch. Phys. Med. Rehabil, Vol. 91, 2010, pp. 1-8 e1. 
4. La lit h a r a t n e, T. D., K. T e r a m o t o, Y. H a y a s h i, K. K i g u c h i. Towards Hybrid EEGEMG-Based Control Approaches to Be Used in Bio-Robotics Applications: Current Status, Challenges and Future Directions. - Paladyn, Journal of Behavioral Robotics, Vol. 4, December 2013, Issue 2, pp. 147-154.

5. Manolova, A., G. Ts enov, V. Laz arova, N. Neshov. Combined EEG and EMG Fatigue Measurement Framework with Application to Hybrid Brain-Computer Interface. In: International Black Sea Conference on Communications and Networking (BlackSeaCom), Sofia, Bulgaria, 2016 (In print).

6. Colorado State University, Brain-Computer Interfaces Laboratory. http://www.cs.colostate.edu/eeg/

7. Sh i r a i, A. S., M. H a s s i b, N. He nze, A. S ch mid t, K. Kunze. What's on Your Mind?: Mental Task Awareness Using Single Electrode Brain Computer Interfaces. - In: Proc. of 5th Augmented Human International Conference, 2014, pp. 45.

8. B a s cil, M. S., A. Y. Tesneli, T. Feyzullah. Multi-Channel EEG Signal Feature Extraction and Pattern Recognition on Horizontal Mental Imagination Task of 1-D Cursor Movement for Brain Computer Interface. Australasian College of Physical Scientists and Engineers in Medicine, 2015.

9. Mou st a f a, M., A. G a d. Classification of Mental Tasks Using Support Vector Machine Based on Linear Predictive Coding and New Mother Wavelet Transform. - In: Proc. of International Conference on Biomedical Engineering and Computational Technologies (SIBIRCON'15), 2015.

10. Harih aran, M., V. Vije an, R. S indhu, P. Divakar, A. S a idatul, S. Yaacob. Classification of Mental Tasks Using Stockwell Transform. - Journal Computers and Electrical Engineering Archive, Vol. 40, July 2014, Issue 5, pp. 1741-1749.

11. Z h a n g, Y., B. L i u, X. J i, D. H u a n g. Classification of EEG Signals Based on Autoregressive Model and Wavelet Packet Decomposition. - Neural Processing Letters, 2017, Issue 2.

12. Lawhern, V., W. D. Hairston, K. Mc Dowell, M. We sterfield, K. Robbins. Detection and Classification of Subject-Generated Artifacts in EEG Signals Using Autoregressive Models. - J. Neurosci Methods, Vol. 208, 2012, pp. 181-189.

13. $\mathrm{H}$ u, D., W. Li, X. C h e n. Feature Extraction of Motor Imagery EEG Signals Based on Wavelet Packet Decomposition. - In: Proc. of IEEE/ICME International Conference on ComplexMedical Engineering (CME'11), 2011, pp. 694-697.

14. Ubeyli, D. Analysis of EEG Signals by Combining Eigenvector Methods and Multiclass Support Vector Machines. - Computers in Biology and Medicine, Vol. 38, 2008, No 1, pp. 14-22.

15. Chang, C.-C., C.-J. Lin. LibSVM: A Library for Support Vector Machines. - ACM Transactions on Intelligent Systems and Technology, Vol. 2, 2011, pp. 27:1-27:27. http://www.csie.ntu.edu.tw/ cjlin/libsvm

16. S c holk o p f, B., A. J. S m o l a. Learning with Kernels. MIT Press, 2002.

17. Kuremoto, T., Y. B aba, M. Ob a y s hi, S. Mabu, K. Kob a y s hi. To Extraction the Feature of EEG Signals for Mental Task Recognition. - In: Proc. of 54th Annual Conference of the Society of Instrument and Control Engineers of Japan (SICE'15), 2015, Hangzhou, pp. 264-269.

18. Li a n g, N., P. S a r a t c h a n d r a n, G. H u a n g, N. S u n d a r a r a j a n. Classification of Mental Tasks from EEG Signals Using Extreme Learning Machine. - International Journal of Neural Systems, Vol. 16, 2006, No 1, pp. 29-38. 